\title{
Determination of variability in serum low density lipoprotein cholesterol response to the replacement of dietary saturated fat with unsaturated fat, in the Reading, Imperial, Surrey Saturated fat Cholesterol Intervention ('RISSCI') project
}

\author{
A. Koutsos ${ }^{1}$, R. Antoni ${ }^{2}$, E. Ozen ${ }^{1}$, G. Wong ${ }^{1}$, L. Sellem ${ }^{1}$, L. Jin ${ }^{1}$, H. Ayyad ${ }^{2}$, N. Jackson ${ }^{2}$, \\ B. A. Fielding ${ }^{2}$, M. D. Robertson ${ }^{2}$, K. G. Jackson ${ }^{1}$, J. A. Lovegrove ${ }^{1}$ and B. A. Griffin ${ }^{2}$ \\ ${ }^{1}$ Hugh Sinclair Unit of Human Nutrition, Department of Nutritional Sciences, University of Reading, RG6 6AP and \\ ${ }^{2}$ Department of Nutritional Sciences, University of Surrey, GU2 $7 W G$
}

Reducing serum low density lipoprotein-cholesterol (LDL-C) by lowering the intake of saturated fatty acids (SFA) to no more than $10 \%$ of total energy, remains the mainstay of dietary guidelines to prevent cardiovascular diseases ${ }^{(1)}$. However, there is marked interindividual variation in the serum LDL-C response to the lowering of SFA. If the variability of this response exceeds the potential reduction in LDL-C, then this may limit the usefulness of this recommendation in the UK population. The overall aim of the RISSCI project is to investigate the mechansims underlying variation in serum LDL-C to dietary SFA, to inform more personalised dietary approaches. To achieve this aim, a study (RISSCI-1) was designed to reproduce the variation in serum LDL-C seen in large dietary intervention trials ${ }^{(2,3)}$. Participants showing hyper and hypo-responsiveness to a reduction in SFA could then be selected and retained for further metabolic investigations (RISCCI-2).

In RISSCI-1, healthy men were recruited at the Universities of Reading and Surrey ( $\mathrm{n}=109$, mean age 48 (range 30-65 years), mean BMI 25.2 (range $19.1-33.3 \mathrm{~kg} / \mathrm{m}^{2}$ ), and were asked to follow a high SFA diet (18\% total energy) for 4 weeks, and then a diet of lower SFA content, equivalent to that of National dietary guideline ( $\leq 10 \%$ total energy) for a further 4 weeks. Dietary SFA was replaced with mono and polyunsaturated fatty acids (MUFA and PUFA) using a food exchange model ${ }^{(4)}$. The diets were iso-energetic, and dietary intake was determined by 4-day diet diaries ( $0,4,8$ weeks). Serum total cholesterol (TC), triacylglycerol (TAG) and HDL-cholesterol (HDL-C) were measured by commercially available colorimetric assays, and LDL-C calculated by the Friedewald formula, at baseline, post-high SFA diet, and post-lower SFA diet.

Replacement of dietary SFA with MUFA and PUFA was achieved with good compliance ${ }^{(4)}$, no effect on BMI, and produced significant reductions in total serum TC and LDL-C of $0.64(12 \%)$ and $0.5 \mathrm{mmol} / \mathrm{L}(15 \%)$, respectively, within 4 weeks (High vs Lower SFA). Inter-individual variation in the change in serum LDL-C ranged from $-40 \%(-1.2 \mathrm{mmo} / \mathrm{L})$ to $+20 \%(3.2 \mathrm{mmol} / \mathrm{L})$, and was of a magnitude previously observed in longer-term intervention trials ${ }^{(2,3)}$.

\begin{tabular}{|l|c|c|c|c|c|c|c|}
\hline $\begin{array}{c}\text { Serum lipids } \\
\text { (mmol/L) }\end{array}$ & \multicolumn{2}{|c|}{ Baseline } & \multicolumn{2}{c|}{ High-SFA } & \multicolumn{2}{c|}{ Lower-SFA } & P-value \\
\cline { 2 - 8 } & mean & SD & mean & SD & mean & SD & \\
\hline TC & $5.15^{\mathrm{a}}$ & 1.01 & $5.41^{\mathrm{a}}$ & 0.90 & $4.77^{\mathrm{b}}$ & 0.90 & $<0.001$ \\
\hline LDL-C & $3.19^{\mathrm{a}}$ & 0.88 & $3.37^{\mathrm{a}}$ & 0.82 & $2.87^{\mathrm{b}}$ & 0.79 & $<0.001$ \\
\hline HDL-C & 1.42 & 0.33 & 1.49 & 0.37 & 1.37 & 0.32 & 0.055 \\
\hline TAG & 1.37 & 0.32 & 1.23 & 0.61 & 1.15 & 0.57 & $0.359^{\star}$ \\
\hline
\end{tabular}

P-values by one-way
ANOVA with post-hoc
Tukey's pairwise
comparison. Different
letters denote
significance (`Log-
transformed data).

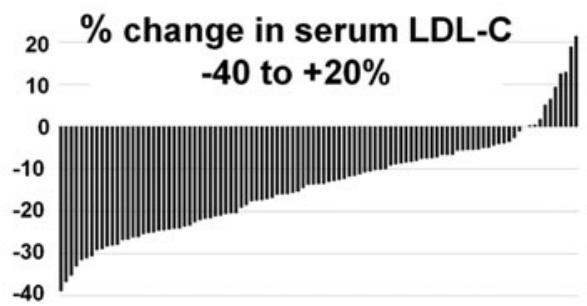

Fig. 1.

These findings support the effectiveness of the dietary guideline to replace SFA with MUFA and PUFA to reduce serum LDL-C, in a short-term intervention. They also demonstrate large inter-individual variation in serum LDL-C, which will allow further investigation of the metabolic and/or genetic origins of this phenomenon in RISCCI-2.

Biotechnology \& Biological Sciences Research Council Grant No. BB/P010245/1

1. Scientific Advisory Committee on Nutrition (2019) Saturated Fats and Health Report: https://www.gov.uk/government/publications/ saturated-fats-and-health-sacn-report.Google Scholar.

2. Jebb SA, Lovegrove JA, Griffin BA et al. (2010) Am J Clin Nutr 92, 748-58.

3. Vafeiadou K, Weech M, Altowaijri H et al. (2015) Am J Clin Nutr 102, 40-8.

4. Antoni R, Sellem L, Koutsos A et al. (2019) Proc Nutr Soc 78, OCE1. 